\title{
BMJ Open The accuracy of the general practitioner's sense of alarm when confronted with dyspnoea and/or thoracic pain: protocol for a prospective observational study
}

\author{
Marie Barais, ${ }^{1}$ Pierre Barraine, ${ }^{1}$ Florie Scouarnec, ${ }^{1}$ Anne Sophie Mauduit, ${ }^{1}$ \\ Bernard Le Floc'h, ${ }^{1}$ Paul Van Royen, ${ }^{2}$ Claire Liétard, ${ }^{3}$ Erik Stolper ${ }^{2,4}$
}

To cite: Barais $M$, Barraine $P$, Scouarnec F, et al. The accuracy of the general practitioner's sense of alarm when confronted with dyspnoea and/or thoracic pain: protocol for a prospective observational study. BMJ Open 2015;5: e006810. doi:10.1136/ bmjopen-2014-006810

- Prepublication history for this paper is available online. To view these files please visit the journal online (http://dx.doi.org/10.1136/ bmjopen-2014-006810).

Received 2 October 2014 Revised 6 February 2015 Accepted 10 February 2015

CrossMark

For numbered affiliations see end of article.

Correspondence to Dr Marie Barais; marie.barais@gmail.com

\section{ABSTRACT}

Introduction: Dyspnoea and chest pain are signs shared with multiple pathologies ranging from the benign to life-threatening diseases. Gut feelings such as the sense of alarm and the sense of reassurance are known to play a substantial role in the diagnostic reasoning of general practitioners (GPs). A Gut Feelings Questionnaire (GFQ) has been validated to measure the GP's sense of alarm. A French version of the GFQ is available following a linguistic validation procedure. The aim of the study is to calculate the diagnostic test accuracy of a GP's sense of alarm when confronted with dyspnoea and chest pain.

Methods and analysis: Prospective observational study. Patients aged between 18 and 80 years, consulting their GP for dyspnoea and/or thoracic pain will be considered for enrolment in the study. These GPs will have to complete the questionnaire immediately after the consultation for dyspnoea and/or thoracic pain. The follow-up and the final diagnosis will be collected 4 weeks later by phone contact with the GP or with the patient if their GP has no information. Life-threatening and non-life-threatening diseases have previously been defined according to the pathologies or symptoms in the (ICPC2) International Collegiate Programming Contest classification. Members of the research team, blinded to the actual outcomes shown on the index questionnaire, will judge each case in turn and will, by consensus, classify the expected outcomes as either life-threatening or non-life-threatening diseases. The sensitivity, the specificity, the positive and negative likelihood ratio of the sense of alarm will be calculated from the constructed contingency table.

Ethics and dissemination: This study was approved by the ethical committee of the University de Bretagne Occidentale. A written informed consent form will be signed and dated by GPs and patients at the beginning of the study. The results will be published in due course.

\section{INTRODUCTION}

Dyspnoea and thoracic pain are signs indicating multiple pathologies from the benign to

\section{Strengths and limitations of this study}

- This is the first study aiming to define the diagnostic accuracy of the sense of alarm when applied to dyspnoea and thoracic pain.

- The setting of this study is of major importance: general practitioners (GPs) will fill in the Gut Feelings Questionnaire during their decisionmaking process

- A limitation of the study is a potential sample selection bias by a potential selection of serious cases, when the participating GPs are not well instructed about the broad inclusion criteria for thoracic pain and dyspnoea.

life-threatening pulmonary embolism or cardiac diseases. General practitioners (GPs) are sometimes torn between missing a patient with a hypothetical life-threatening disease and referring too many patients for harmful and costly investigations. ${ }^{1}$

In 2009, the concept of gut feelings in general practice was described, by means of a qualitative study, as a sense of alarm and a sense of reassurance. ${ }^{2}$ The sense of alarm is an uncomfortable feeling, experienced by physicians, that something does not fit in a patient's clinical presentation although they have found no specific indications. The sense of alarm activates the diagnostic process and induces the doctor to initiate specific management to prevent serious health problems. ${ }^{2}$ It was considered to play a substantial role in the diagnostic reasoning of GPs. ${ }^{3}$ A Dutch Gut Feelings Questionnaire (GFQ) was created from the consensus criteria for gut feelings and validated by a construct validation procedure using case vignettes. The internal consistency of the GFQ proved to be high (Cronbach's $\alpha=0.91$ ), the $\kappa$ with quadratic weighting was moderate 
to $\operatorname{good}(0.62,95 \%$ CI 0.55 to 0.69$) .{ }^{4}$ A linguistic validation procedure was performed to obtain an English version of the questionnaire. ${ }^{4}$

A linguistic validation procedure produced a French version of the GFQ using the same procedure as the Dutch team, ${ }^{56}$ as described in the article presenting the questionnaire. ${ }^{4}$

\section{Limitations of the existing literature}

In the Netherlands, GPs have regularly been blamed, in medical disciplinary tribunals, for failing to respond to this sense of alarm or even because of a lack of it. ${ }^{7}$ This alarm bell should have signalled a dangerous situation in the clinical cases in question: the GP should have reacted in order to prevent an error. The sense of alarm was seen as a means of guaranteeing optimal care. It would have acted as an error-prevention tool. ${ }^{8}$ Taking the wrong decision can lead to serious consequences, both for the patient's health and, at a judicial level, for the doctor concerned.

The accuracy of gut feelings as a diagnostic test was studied in the field of paediatrics. ${ }^{9}$ Gut feelings had a higher specificity than clinical impression in the context of serious infection in children in primary care. The authors recommended that gut feelings should not be ignored but used as a red warning flag. ${ }^{9}$ Gut feeling was considered a fairly accurate tool in the case of chest pain. ${ }^{10}$ GPs interviewed in a qualitative study explained how they had followed their sense of alarm when they correctly diagnosed pulmonary embolism. ${ }^{11}$ They used the sense of alarm as a tool for preventing the diagnostic error of missing a pulmonary embolism. To our knowledge, data are scarce on the diagnostic accuracy of the sense of alarm in primary care, especially for patients with dyspnoea and chest pain.

\section{Objective}

The aim of the study is to calculate the diagnostic test accuracy of the sense of alarm when applied to dyspnoea and chest pain, using the GFQ. ${ }^{4}$ The sensitivity and specificity will be indicated by means of a contingency table, using horizontal lines to indicate life-threatening and non-life-threatening pathologies (in accordance with dyspnoea and chest pain) and vertical lines to indicate the presence or absence of the sense of alarm. This research design will enable us to assess the extent to which GPs can trust their sense of alarm when confronted with dyspnoea and/or thoracic pain.

\section{METHODS AND ANALYSIS}

We will be implementing a prospective observational study using the French version of the GFQ.

\section{Participants}

Patients aged between 18 and 80 years, consulting their GPs for dyspnoea and/or thoracic pain, will be considered for enrolment in the study. GPs involved in the
General Practice Faculty of Brest University will be selected for the study. The participants will not be directly incentivised to take part. In order to increase their involvement, the trainee assisting them at the surgery will be responsible for communicating the information to patients. In France, patients are not used to being involved in research programmes at their GP's surgery. Informing them of the study design and answering their questions are too time-consuming for French GPs. The trainee's role will be to facilitate this information phase. Consecutive patients, for whom dyspnoea and/or thoracic pain are the reason for contact, will be enrolled over a period of 12 weeks. Dyspnoea is defined as difficult or laboured breathing, according to the definition in Medical Subject Headings (MeSH), the National Library of Medicine controlled vocabulary thesaurus used for indexing PubMed citations. Chest pain is defined as pressure, burning or numbness in the chest (MeSH definition). The GPs will be informed directly of the results of the study.

Non-inclusion criteria are: patients in palliative care and patients known to have coronary heart disease. Patients known to have pulmonary diseases are not excluded because of the possibility of the coexistence of pulmonary embolism or secondary infection and other pulmonary pathologies such as chronic obstructive pulmonary disease in the same patient.

In order to limit selection bias in cases, the GPs and trainees will be trained to detect all cases and to not focus solely on the serious ones presented at the surgery. The GPs and trainees will be shown a Powerpoint presentation describing the $\mathrm{MeSH}$ definition of thoracic pain and dyspnoea, including case vignettes. Inclusion and non-inclusion criteria will figure on the back of each questionnaire.

\section{Sample size}

The size of the sample will be estimated according to the following data. The incidence of the sense of alarm was $7 \%$ for the respiratory International Collegiate Programming Contest (ICPC) code chapter and 15\% for the circulatory ICPC code chapter in the first Dutch study. ${ }^{12}$ The prevalence of consultations for dyspnoea in France in primary care is $1.77 \%$ and $1.51 \%$ for thoracic pain. ${ }^{13}$ We defined our initial population as 40 volunteer GPs, each following up, on average, 800 patients in their practice. We included a physician level and a patient level in our calculation. The number of cases required for a power of $80 \%$ and an error rate of $5 \%$ is 211 for thoracic pain with 34 GPs and 123 for dyspnoea with 31 GPs. Taking into account the Lasagna effect, ${ }^{14}$ we estimated seven cases of thoracic pain per GP and four cases of dyspnoea per GP. Epi Info V.6.04 software will be used to perform the analysis.

\section{Data collection}

The final diagnosis will be collected 4 weeks later: by phone contact with the GP to find out how the patient's 
condition has progressed; or by phone contact with the patient if the GP has no information. One researcher from the team will collect follow-up data and gather information on the way used to achieve final diagnosis. We will not include specific analysis on the accuracy of the test used to establish this final diagnosis. Life-threatening and non-life-threatening diseases have previously been defined according to the pathologies or symptoms in the ICPC2 classification. This document was formulated, following a consensus procedure, by a group of experts on the topic. Members of the current research team, blinded to the actual outcomes shown on the index questionnaire, will judge each case in turn and will, by consensus, classify the expected outcomes as either life-threatening or non-life-threatening diseases. The study will take place between 30 January and 31 July 2015.

\section{Data analysis}

The GFQ consists of 10 items. The first five items in the questionnaire are derived from the consensus statements from the gut feelings concept, which describes the sense of reassurance (item 1) and the sense of alarm (items 2-5). ${ }^{2}$ The items are rated using a five-point Likert scale ranging from completely disagree to completely agree. Items numbered $6,7,8$ and 9 relate to the patient's care. The 10th item assesses whether the patient's case elicited a gut feeling (a sense of reassurance or a sense of alarm) or whether it is impossible for the respondent to say or even whether a gut feeling is not applicable. A sense of alarm will be considered as present when the answer to item 10 indicates a sense of alarm or when the answer to item 10 indicates that it is not applicable and at least one of the scores of items 2-5 is higher than $3 / 5$. A sense of alarm is considered as not present when the answer to item 10 indicates either a sense of reassurance or when the answer to item 10 indicates that it is not applicable and the score for item 1 is higher than $3 / 5$. The contingency table will be composed of horizontal lines to indicate a life-threatening or a non-life-threatening pathology (in accordance with dyspnoea and chest pain) and vertical lines to indicate the presence or absence of the sense of alarm. The sensitivity, the specificity, the positive and negative likelihood ratio (LR+ and LR-) will be calculated from the constructed contingency table.

\section{DISCUSSION}

To the best of our knowledge, this is the first study aiming to define the diagnostic accuracy of the sense of alarm when applied to dyspnoea and thoracic pain using the validated GFQ. The setting of this study is of major importance: GPs will fill in the GFQ during their decision-making process. We are providing quantitative data from daily practice situations.

One limit of the study may be the selection of cases. The definitions of thoracic pain and dyspnoea from
MeSH terms are broad and GPs may focus only on what they think are serious cases. We will, of necessity, take inclusion procedures into account during the presentation of the study, and emphasise the definitions of the inclusion criteria, thoracic pain and dyspnoea.

It is more likely that patients will be referred in cases where GPs experience a sense of alarm than in cases where they do not experience any gut feeling. The likelihood of being admitted for a life-threatening pathology may be influenced by additional testing undertaken during secondary care. The analysis of the answers to the questionnaire will be undertaken as soon as the completed questionnaires have been received in order to minimise interpretation bias.

Another limit is related to the transferability of the results to other European countries. French GPs do not use blood point of care testing to help their decisionmaking. D-dimer test and troponin test cannot be carried out at the GP's office, they must be performed in a laboratory.

\section{DISSEMINATION}

The GPs will sign a written consent after receiving information, by email and also by post. Patients will sign a written consent after information has been delivered orally, as well as in writing, by a trainee.

The findings of this study complete the description of the sense of alarm by contributing an essential quantitative component. We will ensure that the results are widely disseminated through publication in open access journals as well as through conference presentations.

\section{Author affiliations}

${ }^{1}$ ERCR SPURBO, Department of General Practice, Univeristé de Bretagne Occidentale, Brest, France

${ }^{2}$ Department of Primary and Interdisciplinary Care, University of Antwerp, Antwerp, Belgium

${ }^{3}$ ERCR SPURBO, Public Health Department, Université de Bretagne

Occidentale, Brest, France

${ }^{4}$ Department of General Practice, Faculty of Health, Medicine and Life Sciences, Caphri School for Public Health and Primary Care, Maastricht University, Maastricht, The Netherlands

Acknowledgements The authors would like to thank Alex Gillman, who provided medical writing services on behalf of the Collège Brestois des Généralistes Enseignants; Sébastien de Villèle, Jean Yves Le Disez and Helen McCombie-Boudry from the Bureau de Traduction Universitaire Université de Bretagne Occidentale; and Jean Sébastien Cadwallader, who provided the translations for the linguistic validation procedure.

Contributors MB, PB, BLF, PVR, CL and ES conceived the study, participated in its design and coordination and helped to draft the manuscript. $C L$ performed the statistical analysis. FS and ASM carried out the linguistic validation procedure and supervised the nominal group. All authors read and approved the final manuscript.

Competing interests None.

\section{Patient consent Obtained.}

Ethics approval This study was approved by the ethical committee of the University de Bretagne Occidentale and declared to the French data protection authority (CNIL) number 1637307 v 0 (12-12-2012). 
Provenance and peer review Not commissioned; externally peer reviewed.

Open Access This is an Open Access article distributed in accordance with the Creative Commons Attribution Non Commercial (CC BY-NC 4.0) license, which permits others to distribute, remix, adapt, build upon this work noncommercially, and license their derivative works on different terms, provided the original work is properly cited and the use is non-commercial. See: http:// creativecommons.org/licenses/by-nc/4.0/

\section{REFERENCES}

1. Pineda LA, Hathwar VS, Grant BJ. Clinical suspicion of fatal pulmonary embolism. Chest 2001:120:791-5.

2. Stolper CF, Van Royen P, Van de Wiel MWJ, et al. Consensus on gut feelings in general practice. BMC Fam Pract 2009;10:66.

3. Stolper CF, Van de Wiel MWJ, Van Royen P, et al. Gut feelings as a third track in general practitioners' diagnostic reasoning. J Gen Intern Med 2011;26:197-203.

4. Stolper CF, Van de Wiel MWJ, De Vet HC, et al. Family physicians' diagnostic gut feelings are measurable: construct validation of a questionnaire. BMC Fam Pract 2013;14:1.

5. Acquadro $\mathrm{C}$, Conway K, Hareendran A, et al. Literature review of methods to translate health-related quality of life questionnaires for use in multinational clinical trials. Value Heal J Int Soc Pharmacoeconomics Outcomes Res 2008;11:509-21.
6. Acquadro C, Conway K, Giroudet C, et al. Linguistic validation manual for patient-reported outcomes (PRO) instruments. Lyon: MAPI Research Institute, 2004.

7. Stolper CF, Legemaate J, Dinant GJ. How do disciplinary tribunals evaluate the "gut feelings" of doctors? An analysis of Dutch tribunal decisions, 2000-2008. J Law Med 2010;18:68-75.

8. Reason JT. Human error. Cambridge University Press, 1990. http:// books.google.com/books?id=WJL8NZc8IZ8C

9. Van den Bruel A, Thompson M, Buntinx F, et al. Clinicians' gut feeling about serious infections in children: observational study. $B M J$ 2012;345:e6144.

10. Buntinx F, Truyen J, Embrechts P, et al. Chest pain: an evaluation of the initial diagnosis made by 25 Flemish general practitioners. Fam Pract 1991;8:121-4

11. Barais M, Morio N, Cuzon Breton A, et al. "I can't find anything wrong: it must be a pulmonary embolism": diagnosing suspected pulmonary embolism in primary care, a qualitative study. PLOS ONE 2014;9:e98112.

12. Stolper CF. Gut Feelings in General Practice. PhD thesis. Maastricht University, 2010

13. Letrilliart L, Supper I, Schuers M, et al. ECOGEN: étude des Éléments de la COnsultation en médecine GENérale. exercer 2014:114:148-57.

14. Van der Wouden JC, Blankenstein $\mathrm{AH}$, Huibers MJH, et al. Survey among 78 studies showed that Lasagna's law holds in Dutch primary care research. J Clin Epidemiol 2007;60:819-24. 